\section{Colonial rule}

\section{Gene E. Robinson}

Bees as Superorganisms: An Evolutionary Reality. By Robin F. A. Moritz and Edward E. Southwick. Springer: 1992. Pp. 411. DM195, \$129, £77.

THE superorganism is back, revived primarily by $\mathrm{E}$. O. Wilson in the mid-1980s, after more than a 20-year absence, to highlight the importance of wedding reductionist analyses of individual insect colony members with a holistic appreciation of the colony. Recently others (D. S. Wilson and E. Sober; T. Seeley) have gone further, asserting that the superorganism is not only a useful metaphor, but a real biological entity. This is also Robin Moritz and Edward Southwick's thesis. One unintended lesson of their book, which focuses on honey bees, is that considerable problems emerge when attempting to move the superorganism from metaphor to reality.

One problem arises when interpretations of colony biology are forced to fit the biology of individual organisms. Moritz and Southwick sometimes resist this temptation, for example when describing how swarming in bees is not genetically equivalent to budding. But they succumb at other times, to erroneous or confusing effect. For example, they conclude that bee colonies sleep, on the basis of only W. Kaiser's suggestive evidence that individual worker bees show sleep-like states of inactivity. M. Lindauer's exhaustive observations, however, have shown that brood rearing and some other tasks are performed around the clock; for such activities a more apt analogy would be E. O. Wilson's "colony as a factory" (with bees working day or night shifts, perhaps). Also, bound by the strictures of this construct, the authors labour to show how the superorganism can die (when the queen dies or leaves with a swarm) while the colony lives on (with a replacement queen). Perhaps this theme, which has important implications for sociogenetics, would be better illuminated by a "colony as a corporation" analogy (the faces may change, but the brand stays the same ....).

A second problem is that it is difficult to define what a superorganism really is, as the authors' idiosyncratic roster reveals. We read that naked mole rats might be superorganisms; but Polistes wasps, to which these fascinating eusocial mammals have often been compared, are not even considered. Even more baffling is the omission of Polybia and other swarm-founding wasps, which in some ways display honey bee-like social complexity. These problems indi- cate that the typological perpective taken here is not the most effective one for understanding the evolution and mechanisms of colony function. An alternative approach that is proving to be productive is to invoke a heuristic metaphor to help analyse a specific feature of colony biology, such as Southwick's "colony as a homeotherm" to describe thermoregulation, or S. Camazine's "colony as an automaton" to describe nest organization.

The book does make a strong case for the idea that natural selection at the level of the colony can be a potent influence on the evolution of both individual and colony traits, an insight that originated from Darwin. It deals comprehensively with only colony biology (as was intended), and social-insect biologists will appreciate the encyclopaedic literature review. Most aspects of the biology of the individual bee are covered only superficially and important areas such as neuroethology are virtually ignored. There are reasonably balanced treatments of several controversial or new topics, including kin recognition and the genetics of division of labour. The extensive coverage of bee genetics (Moritz's own area of expertise) is useful because this topic has received much recent empirical and theoretical attention. But because of its misinterpretations, errors of fact and attribution, and numerous typographical errors, the book will not compete with Tom Seeley's Honey Bee Ecology (Princeton University Press, 1985) and Mark Winston's The Biology of the Honey Bee (Harvard University Press, 1987) as the most useful and enjoyable treatises on the honey bee (individual and colony) for students or generalists.

\section{Gene E. Robinson is in the Department of} Entomology, University of Illinois, Urbana, Illinois 61801, USA.

- Yosiaki Itô's Behaviour and Social Evolution of Wasps: The Communal Aggregation Hypothesis has just been published in Oxford University Press's Series in Ecology and Evolution. The author argues that kin selection has been over-emphasized as an evolutionary explanation of sociality. $£ 30, \$ 61$ (hbk); £28, \$13.50 (pbk)

\title{
Forbidden symmetries
}

\section{Walter Steurer}

Quasicrystals: A Primer. By C. Janot. Oxford University Press: 1992. Pp. 320. $£ 42.50, \$ 65$.

"GooD order is the foundation of all good things", wrote Edmund Burke. For many years, crystallographers were sure that fivefold symmetry and crystalline order were incompatible. But in 1984, D. Shechtman's discovery of grains with icosahedral diffraction symmetry in a rapidly cooled aluminium-manganese alloy opened heated debates about the existence of this new ordering state of matter. The feverish activity in the study of structure and properties of these phases, later called quasicrystals, is reflected in more than 2,000 publications so far: how noncrystallographic symmetry and quasiperiodic translational order combine to give perfectly ordered structures is now essentially understood. What quasicrystals are good for is hinted at by Janot: "For quasicrystals, the age of effectiveness has already started to overlap the dreaming period". Indeed, quasicrystals are now finding uses as surface coatings, for instance for non-stick frying pans.

An easily readable textbook to guide a wide range of readers through this enormous amount of diverse information has long been needed. In Quasicrystals, Janot presents clearly the fundamentals of quasicrystal research, starting with an introduction to crystallography that cov- ers geometrical properties and construction methods of quasiperiodic tilings. In the following experimental section, he describes the preparation and characterization of real quasicrystals, and includes some wonderful photographs of quasicrystals with dodecahedral or triacontahedral growth morphology. An answer to the question "where are the atoms?" can be found in the chapters on quasicrystal structure analysis. The theory and practice of quasicrystallography's most powerful tool, the high-dimensional embedding method, is presented using the example of icosahedral $\mathrm{Al}-\mathrm{Cu}-\mathrm{Li}$. The strange behaviour of phasons, or phase fluctuations, as well as defects and lattice dynamics, are discussed in the chapters on the physics of quasicrystals.

Janot was one of the pioneers of experimental quasicrystal research, and his authority and influence pervade the book. This sometimes results, however, in a preponderance of his own work on icosahedral quasicrystals at the cost of important contributions by other scientists. And decagonal phases, or twodimensional quasicrystals, gain only a mention.

On the whole, however, the book is an excellent primer for all who are interested in the fascinating field of quasicrystals.

Walter Steurer is in the Institute for Mineralogy, University of Hannover, Welfengarten 1, D-3000 Hannover 1, Germany. 